\title{
Role of retinal image-based counseling in the treatment of peripheral retinal lesions
}

\author{
Ashish Sharma ${ }^{1} \cdot$ Pankaj Dongre $^{1} \cdot$ Keerthi Bellala ${ }^{1} \cdot$ Prahlad Reddy $^{1} \cdot$ Baruch D. Kuppermann $^{2} \cdot$ Francesco Bandello $^{3}$
}

Received: 16 July 2018 / Accepted: 17 July 2018 / Published online: 17 August 2018

(C) The Royal College of Ophthalmologists 2019

The role of peripheral retinal imaging is evolving very fast, especially after advancement of wide-field imaging technology. In a routine clinic, the retina specialist examines the peripheral lesions with the indirect ophthalmoscope, and they verbally explain about the lesion and need of treatment. Most of these peripheral lesions do not cause the visual disturbance. However, they are considered as risk factor for retinal complications [1]. Counseling plays an important role to make asymptomatic patients understand whenever treatment is advised. Several authors have established the decisive role of images-assisted health communication is many areas of medicine [2-5]. However, the role of image-based counseling of patients for peripheral retinal lesions is not well studied. We investigated the role of image-based counseling compared to verbal counseling for the management of peripheral retinal lesions.

The study included all the patients who visited the department of vitreoretina at Lotus Eye Hospital and Institute as a routine screening before refractive surgery from January 2017 to January 2018. The methodology adhered to the tenets of the declaration of Helsinki. Institute's committee on human research has approved the protocol. Informed consent was taken. Retina specialist (A. S.) examined the patients with the indirect ophthalmoscope. Patients who were considered for barrage laser prophylactic treatment after indirect examination were

Ashish Sharma

drashish79@hotmail.com

1 Department of Vitreoretina, Lotus Eye Hospital and Institute, Coimbatore, TN, India

2 Gavin Herbert Eye Institute, University of California, Irvine, Irvine, CA, USA

3 Department of Ophthalmology, Scientific Institute San Raffaele, University Vita-Salute, Milano, Italy randomized with computer-generated random table for verbal (Group A 30 patients, 40 eyes) and image (Fig. 1) assisted counseling (Group B, 30 patients, 40 eyes). Imaging was done with MII Ret Cam (MII Ret Cam Inc, Coimbatore, TN, India). Baseline parameters were similar in both the groups (Table 1). Patient Satisfaction Questionnaire was analyzed and compared between both the groups. Responses to each item were given a 5-point scale ranging from strongly agree (5 points) to strongly disagree (0 points). (Table $2 \mathrm{~A})$

The data collected from the patients were analyzed using SPSS version 16.0 for windows. Descriptive analysis was carried out to exhibit the frequency observations, mean and standard deviation. Independent $t$-test analysis was used to test the significant difference between two groups on selected variables.

The responses of the patient survey $(n=60)$ showed that image-based explanation is superior to the verbal explanation at each level of understanding starting from patients understanding about the disease up to its treatment options and willingness to undergo the procedure. (Table 2B)

As clinicians discover new findings in the peripheral retina with wide-field retinal imaging, the role of right communication regarding the significance of these lesions will increase. The present study is an example of the regular clinic that shows how visual cues can help in patient satisfaction and help them to make treatment decisions. Retinal imaging is fast progressing towards wide-field imaging; we expect clinicians and researchers who are working in this area to monitor patient-centric data along with scientific data.

The study has limitations in terms of small sample size. However, the study does highlight the role of imagebased counseling in peripheral retinal lesions. Further large studies in this area with modalities such as Optos (Optos plc, Dunfermline, UK) can help to understand the importance of wide-field imaging in patient education and counseling. 
Fig. 1 Representative image of the hole and lattice degeneration used for patient counseling

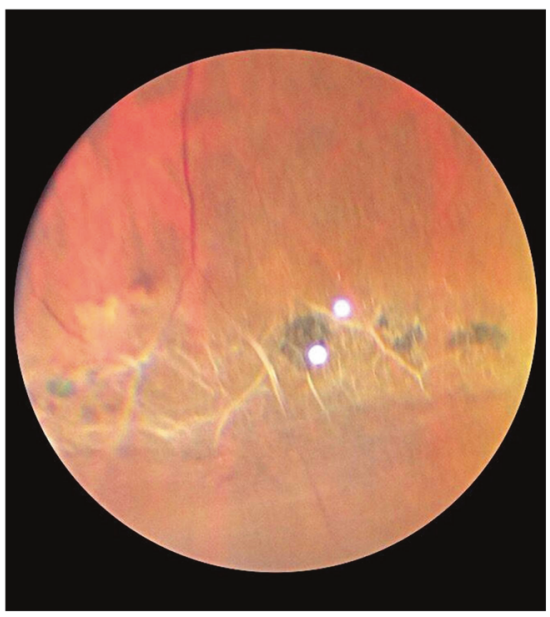

A

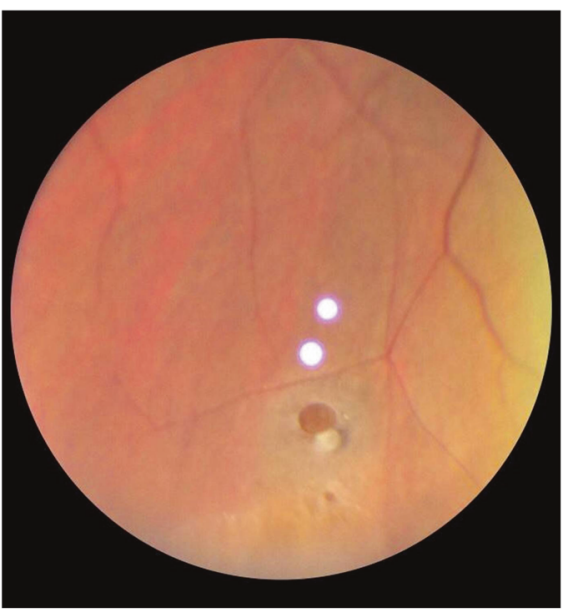

B

Table 1 Comparison of baseline parameters between 2 groups

\begin{tabular}{lllll}
\hline Study variable & Group & $N$ & Data & $P$ Value \\
\hline Age (Mean \pm SD) & A & 30 & $24.16 \pm 4.94$ & $25.36 \pm 5.88$ \\
& B & 30 & 0.76 & 0.39 \\
M/F (Ratio) & A & 30 & 0.87 & 60 \\
Lattice $(\%)$ & B & 30 & 66.6 & 0.59 \\
Retinal hole $(\%)$ & A & 30 & 40 & 0.59 \\
& B & 30 & 33.3 \\
\hline
\end{tabular}

Table 2A Patient Satisfaction Questionnaire (PSQ) with 1-5-point scale

\begin{tabular}{|c|c|c|c|c|c|}
\hline Questions & Strongly disagree & Disagree & Uncertain & Agree & Strongly agree \\
\hline The doctor gave a thorough examination with a head-mount light (Indirect) & 1 & 2 & 3 & 4 & 5 \\
\hline The doctor explained to me about the peripheral retinal problem well & 1 & 2 & 3 & 4 & 5 \\
\hline I completely understood my retinal problem after doctors explanation & 1 & 2 & 3 & 4 & 5 \\
\hline I understood that I need laser treatment for this condition & 1 & 2 & 3 & 4 & 5 \\
\hline I would like to undergo the treatment advised by the doctor & 1 & 2 & 3 & 4 & 5 \\
\hline I would like to take a second opinion before undergoing treatment & 1 & 2 & 3 & 4 & 5 \\
\hline
\end{tabular}

Table 2B Analysis of Mean Patient Satisfaction Questionnaire (PSQ) score between two groups

\begin{tabular}{lll}
\hline Questions & $\begin{array}{l}\text { Verbal explanation mean } \\
\text { score }(n=30)\end{array}$ & $\begin{array}{l}\text { Image-based explanation mean } \\
\text { score }(n=30)\end{array}$ \\
\hline The doctor gave a thorough examination with a head-mount light (Indirect) & 5 & 5 \\
The doctor explained me about the peripheral retinal problem well & $3.8 \pm 0.5$ & $4.7 \pm 0.4$ \\
I completely understood my retinal problem after doctors explanation & $2.3 \pm 0.5$ & $4 \pm 0.6$ \\
I understood that I need laser treatment for this condition & $2.3 \pm 0.5$ & $4 \pm 0.6$ \\
I would like to undergo the treatment advised by the doctor & $3 \pm 0.6$ & $3.9 \pm 0.8$ \\
I would like to take a second opinion before undergoing treatment & $3.2 \pm 0.8$ & $2.2 \pm 1.1$ \\
\hline
\end{tabular}




\section{Compliance with ethical standards}

Conflict of interest A.S. is an Innovator of MII Ret Cam. None of the other authors have the conflict of interest with this submission

\section{References}

1. Chan CK, Tarasewicz DG, Lin SG. Relation of pre-LASIK and post-LASIK retinal lesions and retinal examination for LASIK eyes. Br J Ophthalmol. 2005;89:299-301. 2
2. Roger Collier. Educating patients with pictures. CMAJ. 2011;183:e1094.

3. Katz MG, Kripalani S, Weiss BD. Use of pictorial aids in medication instructions: a review of the literature. Am J Health Syst Pharm. 2006;63:2391-7.

4. Chan HK, Hassali MA, Lim CJ, Saleem F, Tan WL. Using pictograms to assist caregivers in liquid medication administration: a systematic review. J Clin Pharm Ther. 2015;40:266-72.

5. Paasche-Orlow MK, Schillinger D, Greene SM, Wagner EH. Health care systems can begin to address the challenge of limited literacy. J Gen Intern Med. 2006;21:884-7. 